\title{
Risk of Clinically Relevant Pharmacokinetic-Based Drug-Drug Interactions with Drugs Approved by the U.S. Food and Drug Administration Between 2013 and 2016 ${ }^{\text {[ }}$
}

\author{
Jingjing Yu, Zhu Zhou, Jessica Tay-Sontheimer, René H. Levy, and Isabelle Ragueneau-Majlessi \\ Department of Pharmaceutics, School of Pharmacy, University of Washington, Seattle, Washington (J.Y., J.T.-S., R.H.L., I.R.-M.); \\ and Department of Pharmaceutics and Medicinal Chemistry, Thomas J. Long School of Pharmacy and Health Sciences, University \\ of the Pacific, Stockton, California (Z.Z.)
}

Received September 29, 2017; accepted March 16, 2018

\section{ABSTRACT}

A total of 103 drugs (including 14 combination drugs) were approved by the U.S. Food and Drug Administration from 2013 to 2016. Pharmacokineticbased drug interaction profiles were analyzed using the University of Washington Drug Interaction Database, and the clinical relevance of these observations was characterized based on information from new drug application reviews. CYP3A was involved in approximately two-thirds of all drug-drug interactions (DDls). Transporters (alone or with enzymes) participated in about half of all interactions, but most of these were weak-to-moderate interactions. When considered as victims, eight new molecular entities (NMEs; cobimetinib, ibrutinib, isavuconazole, ivabradine, naloxegol, paritaprevir, simeprevir, and venetoclax) were identified as sensitive substrates of CYP3A, two NMEs (pirfenidone and tasimelteon) were sensitive substrates of CYP1A2, one NME (dasabuvir) was a sensitive substrate of CYP2C8, one NME (eliglustat) was a sensitive substrate of CYP2D6, and one NME (grazoprevir) was a sensitive substrate of OATP1B1/3 (with changes in exposure greater than 5-fold when coadministered with a strong inhibitor). Approximately $75 \%$ of identified CYP3A substrates were also substrates of P-glycoprotein. As perpetrators, most clinical DDls involved weak-to-moderate inhibition or induction. Only idelalisib showed strong inhibition of CYP3A, and lumacaftor behaved as a strong CYP3A inducer. Among drugs with large changes in exposure ( $\geq 5$-fold), whether as victim or perpetrator, the mostrepresented therapeutic classes were antivirals and oncology drugs, suggesting a significant risk of clinical DDIs in these patient populations.

\section{Introduction}

Pharmacokinetic (PK)-based drug-drug interactions (DDIs) constitute one of the major causes of drug withdrawal from the market in recent decades (Huang et al., 2008). Mechanistic methodologies have been used by the pharmaceutical industry to assess DDI risk during the drugdevelopment process. Currently, these methodologies include evaluation of the potential of a new molecular entity (NME) to affect the metabolism or transport of other drugs and the potential for the new drug's metabolism or transport to be affected by other drugs, with recommended clinical index substrates and specific inhibitors/inducers of drug-metabolizing enzymes (DMEs) or transporters (https://www. fda.gov/Drugs/DevelopmentApprovalProcess/DevelopmentResources/ DrugInteractionsLabeling/ucm093664.htm; Food and Drug Administration, 2012a). Additionally, if an NME is commonly used with another drug in a designated patient population, it is recommended that the DDI risk between the two drugs be evaluated. This review encompasses a detailed analysis of clinical DDIs mediated by DMEs and transporters based on new

https://doi.org/10.1124/dmd.117.078691.

S This article has supplemental material available at dmd.aspetjournals.org. drug applications (NDAs) approved by the U.S. Food and Drug Administration (FDA) from 2013 to 2016. It highlights the main mechanistic findings and discusses their clinical relevance, identifying substrates with varying degrees of sensitivity and inhibitors/inducers with varying potency of DMEs and transporters, and how these findings are reflected in the labeling. These findings will aid in the understanding, predict, and reduce DDI risk and associated adverse reactions in certain patient populations, in which polytherapy is common. Through systematic analysis, this review aimed to provide communications on DDI risk evaluation and management as well as clinical implications to pharmaceutical researchers and health care providers.

\section{Materials and Methods}

This analysis was performed using the University of Washington Drug Interaction Database, a drug interaction and pharmacogenetic (PGx) database (http://www. druginteractioninfo.org). Clinical DDI study results included in this analysis were generated from dedicated DDI clinical trials, PGx studies, as well as physiologically based pharmacokinetics (PBPK) simulations that are used as alternatives to dedicated clinical studies. As in previous publications, mean area under the drug plasma concentration-time curve (AUC) and maximum plasma concentration $\left(C_{\max }\right)$ ratios that are systematically presented by the Drug Interaction Database are the metrics used

ABBREVIATIONS: AUC, area under the drug plasma concentration-time curve; BCRP, breast cancer resistance protein; CNS, central nervous system; DDI, drug-drug interaction; DME, drug-metabolizing enzyme; FDA, Food and Drug Administration; FDC, fixed-dose combination; MRP, multidrug resistance-associated protein; NDA, new drug application; NME, new molecular entity; NTR, narrow therapeutic range; OAT, organic anion transporter; OATP, organic anion transporting polypeptide; OCT, organic cation transporter; P450, cytochrome P450; PBPK, physiologically based pharmacokinetics; P-gp, P-glycoprotein; PGx, pharmacogenetics; PK, pharmacokinetics; UM, ultrarapid metabolizer. 
to evaluate clinical studies. In the present analysis, all positive clinical studies (defined as AUC ratios $\geq 1.25$ for inhibition and $\leq 0.8$ for induction) were analyzed. Because a 2-fold change in drug exposure often triggers dose recommendations, all DDI studies with exposure changes $\geq 2$-fold were highlighted regardless of labeling effects. Also, studies with drug exposure changes of 1.25- to 2-fold and still triggering dose recommendations are presented. In accordance with the FDA classification (FDA, 2012a), NMEs were considered as sensitive or moderate sensitive clinical substrates if they demonstrated maximum AUC ratios of $\geq 5$ or $2-5$, respectively, with strong inhibitors of a given metabolic pathway. Therefore, in this review, the DDI results were presented based on inhibition studies when NMEs were evaluated as substrates, with additional evidence from drug interaction studies using strong inducers. On the other hand, an NME was considered as a strong, moderate, or weak clinical inhibitor or inducer (of a given disposition pathway) when the observed maximum AUC ratio was $\geq 5,2-5$, and $1.25-2$, respectively, for inhibitors, and $\leq 0.2,0.2-0.5$, and $0.5-0.8$, respectively, for inducers, with coadministration of a sensitive clinical substrate.

\section{Results}

From 2013 to 2016, a total of 103 NDAs [including 14 combination drugs, total NMEs $=107$; Supplemental Table 1, with chemical structures presented in Supplemental Table 2 for drugs approved in 2016 and previous publications (Yu et al., 2014, 2016, 2017) for drugs approved from 2013 to 2015] and 32 biologics license applications were approved by the FDA. Because of the different disposition and elimination mechanisms of biologics compared with small molecules and their low risk for PK-based drug interactions, biologics license applications contain few studies relevant to the present analysis and were not included in this review. Among all the NDAs included in the analysis, the most represented therapeutic areas were oncology (21\%) and anti-infective drugs (20\%), followed by central nervous system (CNS) agents (13\%), metabolism disorder/endocrinology drugs (11\%), and cardiovascular drugs (10\%). Among the anti-infective drugs
$(N=21)$, there are 10 antivirals, six antibacterials, four antifungals, and one antiparasitic. Ninety-eight of the 103 NDAs had drug metabolism data and 81 had transporter data available, including in vitro and/or clinical evaluations. NDAs for all years analyzed included extensive in vitro evaluations of drug metabolism profiles ranging from $88 \%$ (in 2013) to $100 \%$ of the NDAs (in 2014). There was an increase in the percentage of NDAs that included assessment of in vitro transport from $73 \%$ to $80 \%$ evaluated between 2013 and 2015 to $93 \%$ evaluated in 2016. In particular, the number of transporter experiments per drug increased dramatically in the past 4 years, from 6 in 2013 to 22 in 2016. The types of transporters evaluated also expanded from 16 (in 2013) to 21 (in 2016). In addition to the nine transporters recommended by the FDA draft guidance (FDA, 2012a) and the International Transporter Consortium white paper (Hillgren et al., 2013), 18 other transporters were assessed in the NDAs. Transporters evaluated in these NDAs included apical sodium-dependent bile acid transporter; bile salt export pump; breast cancer resistance protein (BCRP); multidrug and toxin extrusion proteins 1 and 2-K (MATE1 and MATE2-K); multidrug resistance-associated proteins $1,2,3,4,5$, and 8 (MRP1, MRP2, MRP3, MRP4, MRP5, and MRP8); organic anion transporters 1, 2, 3, and 4 (OAT1, OAT2, OAT3, and OAT4); organic anion transporting polypeptides 1A2, 1B1, 1B3, and 2B1 (OATP1A2, OATP1B1, OATP1B3, and OATP2B1); organic cation transporters 1, 2, and 3 (OCT1, OCT2, OCT3); organic cation/carnitine transporters 1 and 2 (OCTN1 and OCTN2); P-glycoprotein (P-gp); sodium-taurocholate cotransporting polypeptide; and urate transporter 1 (URAT1). Finally, in addition to clinical DDI studies, 16 NDAs presented PGx information, and 16 had PBPK simulation data that directly supported dosing recommendations. An analysis of clinically relevant DDI findings and related in vitro investigations is presented in the following sections. Key DDI findings are summarized in Tables 1-4,

TABLE 1

Inhibition DDIs with AUC ratios $\geq 5$, NME as substrate

Drugs were orally administered unless specified.

\begin{tabular}{|c|c|c|c|c|}
\hline Victim Drug & Inhibitor & Main Enzymes/Transporters Possibly Involved & AUC Ratio & Reference \\
\hline Paritaprevir & Ritonavir & CYP3A, P-gp, BCRP, OATP1B1/3 & 47.43 & FDA $(2014 m)$ \\
\hline Eliglustat & Ketoconazole/paroxetine & CYP3A, CYP2D6 ${ }^{a}$ & 37.85 (PBPK in EMs) & FDA $(2014 c)$ \\
\hline Eliglustat & Paroxetine & CYP2D6 & $28.40(\mathrm{UMs})$ & FDA (2014c) \\
\hline Ibrutinib & Ketoconazole & CYP3A & 23.90 & FDA $(2013 g)$ \\
\hline Eliglustat & Fluconazole/terbinafine & CYP3A, CYP2D6 & $19.31\left(\mathrm{AUC}_{0-24 \mathrm{~h}}, \mathrm{PBPK}\right.$ in EMs$)$ & FDA $(2014 c)$ \\
\hline Grazoprevir & Cyclosporine & OATP1B $1 / 3^{b}$ & $15.25\left(\mathrm{AUC}_{0-24 \mathrm{~h}}\right)$ & FDA (2016d) \\
\hline Grazoprevir & Lopinavir/ritonavir & CYP3A, OATP1B1/3 ${ }^{b}$ & 12.87 & FDA (2016d) \\
\hline Naloxegol & Ketoconazole & $\mathrm{CYP} \mathrm{A}^{a}$ & 12.42 & FDA (2014h) \\
\hline Grazoprevir & Atazanavir/ritonavir & CYP3A, OATP $1 \mathrm{~B} 1 / 3^{b}$ & 10.56 & FDA (2016d) \\
\hline Grazoprevir & Rifampin (i.v.) & OATP1B1/3 & 10.22 & FDA (2016d) \\
\hline Eliglustat & Paroxetine & CYP2D6 & $10.00(\mathrm{EMs})$ & FDA (2014c) \\
\hline Dasabuvir & Gemfibrozil & CYP2C8 & 9.90 & FDA $(2014 m)$ \\
\hline Eliglustat & Ketoconazole/paroxetine & $\mathrm{CYP}^{2} \mathrm{~A}, \mathrm{CYP}^{2} \mathrm{D}^{a}$ & 9.81 (PBPK in IMs) & FDA $(2014 c)$ \\
\hline Ibrutinib & Erythromycin & CYP3A & $8.60(\mathrm{PBPK})$ & FDA $(2013 g)$ \\
\hline Grazoprevir & Rifampin & OATP1B $1 / 3^{b}$ & 8.37 & FDA (2016d) \\
\hline Ivabradine & Josamycin & $\mathrm{CYP}_{3} \mathrm{~A}^{a}$ & 7.70 & FDA $(2015 c)$ \\
\hline Ivabradine & Ketoconazole & $\mathrm{CYP} 3 \mathrm{~A}^{a}$ & 7.70 & FDA $(2015 c)$ \\
\hline Eliglustat & Fluconazole & CYP3A & 7.54 (PBPK in PMs) & FDA $(2014 c)$ \\
\hline Grazoprevir & Darunavir/ritonavir & CYP3A, OATP1B $1 / 3^{b}$ & 7.49 & FDA (2016d) \\
\hline Simeprevir & Ritonavir & $\mathrm{CYP} \mathrm{A}^{a}$ & 7.18 & FDA (2013i) \\
\hline Tasimelteon & Fluvoxamine & CYP1A $2^{c}$ & 6.87 & FDA (2014f) \\
\hline Pirfenidone & Fluvoxamine & CYP1A2 & 6.81 (smokers), 3.97 (nonsmokers) & FDA (2014d) \\
\hline Cobimetinib & Itraconazole & CYP3A ${ }^{a}$ & 6.62 & FDA $(2015 d)$ \\
\hline Simeprevir & Erythromycin & $\mathrm{CYP}_{3} \mathrm{~A}^{a}$ & 6.54 & FDA (2013i) \\
\hline Flibanserin & Fluconazole & CYP3A, CYP2C19 & 6.41 & FDA (2015a) \\
\hline Venetoclax & Ketoconazole & CYP3A, P-gp & 6.40 & FDA (2016e) \\
\hline Eliglustat & Ketoconazole & $\mathrm{CYP} \mathrm{A}^{a}$ & 6.22 (PBPK in PMs) & FDA $(2014 c)$ \\
\hline Ibrutinib & Diltiazem & CYP3A & $5.50(\mathrm{PBPK})$ & FDA (2013g) \\
\hline Isavuconazonium sulfate (prodrug) & Ketoconazole & CYP3A, butyrylcholinesterase & 5.22 & FDA $(2015 \mathrm{e})$ \\
\hline Eliglustat & Paroxetine & CYP2D6 & $5.20(\mathrm{IMs})$ & FDA $(2014 c)$ \\
\hline
\end{tabular}

EM, CYP2D6 extensive metabolizer; IM, CYP2D6 intermediate metabolizer; PM, CYP2D6 poor metabolizer; UM, CYP2D6 ultrarapid metabolizer; i.v., intravenously

${ }^{a}$ Also a substrate of P-gp based on in vitro results; inhibition of P-gp might contribute to the observed interaction.

${ }^{b}$ Also a substrate of P-gp and BCRP based on in vitro results.

${ }^{c}$ Also metabolized by CYP3A, CYP2C9, and CYP2C19; fluvoxamine inhibits these P450s. 
TABLE 2

Induction DDIs with AUC ratios $\leq 0.2$, NME as substrate

\begin{tabular}{|c|c|c|c|}
\hline Victim Drug & Main Enzymes/Transporters Possibly Involved & AUC Ratio & Reference \\
\hline Isavuconazonium sulfate & CYP3A, butyrylcholinesterase & 0.03 & FDA $(2015 \mathrm{e})$ \\
\hline Eliglustat & CYP3A $^{a}$ & 0.04 (PMs) & FDA $(2014 c)$ \\
\hline Flibanserin & CYP3A, CYP2C19 & 0.04 & FDA (2015a) \\
\hline Ibrutinib & $\mathrm{CYP}_{3} \mathrm{~A}^{a}$ & 0.08 (PBPK) & FDA $(2013 g)$ \\
\hline Eliglustat & $\mathrm{CYP} \mathrm{A}^{a}$ & 0.09 (IMs) & FDA $(2014 c)$ \\
\hline Eliglustat & $\mathrm{CYP} \mathrm{A}^{a}$ & 0.10 (EMs) & FDA $(2014 c)$ \\
\hline Naloxegol & $\mathrm{CYP} \mathrm{A}^{a}$ & 0.11 & FDA $(2014 h)$ \\
\hline Olaparib & $\mathrm{CYP} \mathrm{A}^{a}$ & 0.11 & FDA $(2014 \mathrm{~g})$ \\
\hline Rolapitant & CYP3A & 0.12 & FDA (2015o) \\
\hline Suvorexant & CYP3A & 0.12 & FDA $(2014 b)$ \\
\hline Tasimelteon & CYP3A ${ }^{a, b}$ & 0.14 & FDA (2014f) \\
\hline Palbociclib & $\mathrm{CYP} \mathrm{A}^{a}$ & 0.15 & FDA $(2015 h)$ \\
\hline Cobimetinib & $\mathrm{CYP} \mathrm{A}^{a}$ & 0.17 (PBPK) & FDA $(2015 d)$ \\
\hline Grazoprevir & $\mathrm{CYP} \mathrm{A}^{c}$ & 0.17 & FDA (2016d) \\
\hline Velpatasvir & CYP2B6, CYP2C8, CYP3A, P-gp, BCRP & 0.19 & FDA (2016b) \\
\hline Netupitant & CYP3A & 0.20 & FDA $(2014 a)$ \\
\hline
\end{tabular}

EM, extensive metabolizer; IM, intermediate metabolizer; PM, poor metabolizer.

${ }^{a}$ Also a substrate of P-gp based on in vitro results; induction of P-gp might contribute to the observed interaction.

${ }^{b}$ Also metabolized by CYP1A2, CYP2C9, and CYP2C19; rifampin is an inducer of multiple P450s.

${ }^{c}$ Also a substrate of P-gp and BCRP based on in vitro results; induction of P-gp and BCRP might contribute to the observed interaction.

including maximum AUC ratios, enzymes and transporters possibly involved, and overall labeling impact. For each interaction, more detailed information, such as dosing regimen for victim and precipitant drugs, study design, study population, and specific labeling impact, is presented in Supplemental Tables 3-6.

\section{NMEs as Substrates}

Overall, for drugs evaluated as substrates, there were approximately 100 inhibition studies with AUC ratios $\geq 2$ and 50 induction studies with AUC ratios $\leq 0.5$ with concomitant administration of inhibitors and inducers, respectively. Additionally, approximately 30 inhibition studies with AUC ratios of 1.25-2 and 10 induction studies with AUC ratios of $0.5-0.8$ were associated with dose recommendations included in the drug label. A total of 53 NMEs served as victim drugs in these interaction studies. Of these drugs, cancer treatments and antivirals are the dominant therapeutic areas (Figs. 1A and 2A).

DDIs with AUC Changes $\mathbf{2 5}$-fold: Sensitive Clinical Substrates. When NMEs served as victims, 14 drugs were found to have AUC ratios $\geq 5$ when coadministered with strong inhibitors (Supplemental Table 3; Table 1). In terms of therapeutic classes, the most represented area is anti-infective agents $(36 \%)$, including four antivirals and one antifungal, followed by cancer treatments $(N=3,21 \%)$ and CNS agents $(N=2,14 \%)$ (Supplemental Fig. 1A). This pattern of prevalence is consistent among drugs approved from 2013 to 2016.

The highest AUC change was observed with the antiviral paritaprevir, which exhibited a 47.43-fold increase in the presence of ritonavir (100-mg single dose, not an NME), a strong inhibitor of CYP3A (also an inhibitor of multiple transporters). This DDI effect was observed in a fixed-dose combination (FDC) drug (ombitasvir/paritaprevir/ritonavir copackaged with dasabuvir), where paritaprevir is administered at low dose $(100 \mathrm{mg})$ and the role of ritonavir is to increase paritaprevir peak and trough concentrations as well as its overall drug exposure.

Eliglustat, a glucosylceramide synthase inhibitor indicated for the treatment of Gaucher disease, exhibited the second-largest DDI effect, wherein the strong CYP2D6 inhibitor paroxetine $(30 \mathrm{mg}$ once daily for 10 days) significantly increased eliglustat AUC 28.40-fold in CYP2D6 ultrarapid metabolizer subjects. Increases of 10.00- and 5.20-fold were observed in CYP2D6 extensive metabolizers and intermediate metabolizers, respectively, when eliglustat was coadministered with paroxetine. Consistent with these findings, the exposure to eliglustat (100 mg twice daily) was 2.60-fold higher in intermediate metabolizers, 7.80-fold higher in poor metabolizers, and $85.6 \%$ lower in ultrarapid metabolizers compared with CYP2D6 extensive metabolizer subjects. Based on these observations, genetic testing is considered necessary before administering eliglustat, and dose adjustment is needed depending on CYP2D6 polymorphism and/or coadministration with a strong or moderate CYP2D6 inhibitor (FDA, 2014c).

Regarding possible mechanism(s) of these large interactions, significant changes in victim drug exposure could be attributed to one or more of the following DMEs and transporters: CYP1A2, CYP2C8, CYP2D6, CYP3A, BCRP, OATP1B1/3, and P-gp (Supplemental Fig. 1B). CYP3A was involved in two-thirds of the drug interactions, either as a

TABLE 3

Inhibition DDIs with AUC ratios $\geq 5$, NME as inhibitor

Drugs were orally administered.

\begin{tabular}{|c|c|c|c|c|}
\hline Victim Drug & Inhibitor & Main Enzymes/Transporters Possibly Involved & AUC Ratio & Reference \\
\hline Tacrolimus & Ombitasvir, paritaprevir, and ritonavir & CYP3A, P-gp & 85.92 & FDA $(2014 m)$ \\
\hline Tacrolimus & Paritaprevir, dasabuvir, and ritonavir & CYP3A, P-gp & 78.68 & FDA $(2014 m)$ \\
\hline Tacrolimus & Ombitasvir, paritaprevir, dasabuvir, and ritonavir & CYP3A, P-gp & 57.07 & FDA $(2014 m)$ \\
\hline Cyclosporine & Ombitasvir, paritaprevir, dasabuvir, and ritonavir & CYP3A, P-gp & 5.78 & FDA $(2014 \mathrm{~m})$ \\
\hline Midazolam & Idelalisib & CYP3A & 5.15 & FDA $(20140)$ \\
\hline
\end{tabular}


TABLE 4

Induction DDIs with AUC ratios $\leq 0.5, \mathrm{NME}$ as inducer

Drugs were orally administered.

\begin{tabular}{llccc}
\hline Victim Drug & \multicolumn{1}{c}{ Inducer } & Main Enzymes/Transporters Possibly Involved & AUC Ratio & Reference \\
\hline Itraconazole & Ivacaftor and lumacaftor & CYP3A & 0.18 & FDA (2015k) \\
Ivacaftor & Lumacaftor & CYP3A & 0.20 & FDA (2015k) \\
Midazolam & Dabrafenib & CYP3A & 0.26 & FDA \\
\hline
\end{tabular}

main contributor or together with other cytochrome P450s (P450s) or transporters. Eight drugs in this group [cobimetinib, ibrutinib, isavuconazole (the active metabolite of prodrug isavuconazonium sulfate), ivabradine, naloxegol, paritaprevir, simeprevir, and venetoclax] were identified as sensitive clinical substrates of CYP3A, with AUC ratios of $6.62,23.90,5.22,7.70,12.42,47.43,7.18$, and 6.40, respectively, when coadministered with strong CYP3A inhibitors such as itraconazole, ketoconazole, or ritonavir. For cobimetinib, ivabradine, naloxegol, paritaprevir, simeprevir, and venetoclax, contributions of P-gp are possible, as in vitro studies showed that they are all substrates of P-gp (FDA, 2014c,h, 2015c,d), and itraconazole, ketoconazole, and ritonavir are known inhibitors of P-gp (https://www.fda.gov/Drugs/Development ApprovalProcess/DevelopmentResources/DrugInteractionsLabeling/ ucm093664.htm; FDA, 1996). Further, OATP1B1/3 (and possibly BCRP) may also be a factor in the interaction between paritaprevir and ritonavir. Due to a lack of specific inhibitors, it remains challenging to identify the exact contribution of each enzyme or transporter to drug disposition. On the other hand, these eight drugs are also sensitive to induction. Coadministration of the strong CYP3A inducers carbamazepine (for paritaprevir), rifampin (for cobimetinib, ibrutinib, isavuconazole, naloxegol, and venetoclax), or St. John's wort (for ivabradine PBPK simulations) or the moderate inducer efavirenz (for simeprevir) significantly reduced drug exposure by $70 \%-97 \%$, suggesting a reduction in therapeutic efficacy (FDA, 2013i, 2014m, 2015c) (Supplemental Table 4; Table 2). In addition to being substrates of CYP3A, four of these drugs were identified as sensitive clinical substrates of other $\mathrm{P} 450 \mathrm{~s}$ - pirfenidone and tasimelteon of CYP1A2, dasabuvir of CYP2C8, and eliglustat of CYP2D6. The plasma exposure of pirfenidone, tasimelteon, dasabuvir, and eliglustat increased 6.81-, 6.87-, 9.90-, and 28.40-fold, respectively, when coadministered with the strong clinical inhibitors fluvoxamine, gemfibrozil, and paroxetine (FDA, 2014c,d,f,m). In vitro studies showed that tasimelteon is also metabolized by CYP3A (FDA, 2014f), and fluvoxamine is a weak inhibitor of CYP3A (Lam et al., 2003). However, coadministration of ketoconazole (strong CYP3A inhibitor) only slightly increased tasimelteon AUC (by 45\%), suggesting that inhibition of CYP3A-mediated metabolism of tasimelteon by fluvoxamine is negligible (FDA, 2014f). A 6.41-fold increase in flibanserin exposure was observed when coadministered with fluconazole, a strong CYP2C19 inhibitor and a moderate CYP3A inhibitor, whereas a smaller change (4.61-fold) was observed with coadministration of the strong CYP3A inhibitor ketoconazole, suggesting that CYP3A plays a primary role in the disposition of flibanserin with partial contribution from CYP2C19, but flibanserin is not a sensitive substrate of CYP3A (FDA, 2015a).

In addition to metabolism, transporters seem to play an important role in some cases. For example, in vitro studies suggest that grazoprevir is a substrate of OATP1B1/3, BCRP, and P-gp (FDA, 2016f). Grazoprevir exposure was increased 10.22-fold with concomitant administration of intravenous rifampin, a clinical inhibitor of OATP1B1/3, suggesting that grazoprevir is a sensitive clinical substrate of OATP1B1/3. A 15.25-fold increase in grazoprevir AUC was observed when coadministered with the multitransporter inhibitor cyclosporine, suggesting an involvement of BCRP and P-gp in addition to OATP (FDA, 2016f).
Consistent with the large changes in drug exposure observed with these 14 drugs ( 13 identified as sensitive substrates), their product labels included clinical recommendations (contraindicate, avoid, not recommend, or reduce the dose).

DDIs with $2 \leq$ AUC Ratios < 5: Moderate Sensitive Clinical Substrates. A total of 28 drugs (including eight drugs overlapping with the group of AUC ratios $\geq 5$ ) demonstrated AUC increases of 2- to 5-fold when coadministered with inhibitors of enzymes and/or transporters. Detailed DDI data are presented in Supplemental Table 3. The majority of these DDIs were addressed in the product labeling, mostly with a recommendation to avoid coadministration or to reduce the dose. The largest number of drugs in this group are antivirals $(N=8)$, followed by cardiovascular drugs $(N=5)$, CNS agents $(N=5)$, cancer treatments $(N=4)$, and gastrointestinal agents $(N=3)$ (Supplemental Fig. 1C). In brief, among the 32 drug interactions identified in this group, the majority are attributable to inhibition of one enzyme or transporter by strong inhibitors (Supplemental Fig. 1D); therefore, the NMEs are considered moderate sensitive substrates in accordance with the FDA classification and terminology (FDA, 2012a). CYP3A plays a dominant role by mediating two-thirds of the drug interactions. Interestingly, P-gp, BCRP, and OATP1B1/3 are involved in approximately one-third of these interactions either as an individual contributor or together with other transporters or CYP3A (Supplemental Fig. 1D). Changes in victim exposure appeared to be no larger than 3-fold for most interactions.

DDIs with $1.25 \leq$ AUC Ratios $<2$ and Triggering Dose Recommendations. As victims, $21 \mathrm{NMEs}$ (five overlapping with the AUC ratio $\geq 5$ group, three overlapping with the AUC ratios between 2 and 5 group, and one overlapping with both groups) were found to have slight increases of less than 2-fold in their exposure when coadministered with inhibitors; however, label recommendations were triggered due to safety concerns (Supplemental Table 3). In most of these cases, the labels included recommendations to monitor drug exposure and/or patients for increased drug exposure-associated adverse reactions and/or reduce dose. The most represented drug areas are cancer treatments $(N=8)$ and antivirals $(N=4)$ (Supplemental Fig. 1E).

CYP3A was again found to be a significant contributor, mediating more than $60 \%$ of the interactions, partially with contributions from other P450s or P-gp/OATP1B1/3 (Supplemental Fig. 1F). However, CYP3A did not seem to play a primary role in the drug disposition of the following NMEs: dabrafenib, dasabuvir, idelalisib, nintedanib, ospemifene, palbociclib, panobinastat, trabectedin, vilanterol, and vorapaxar.

Different label recommendations were triggered on the basis of different DDI scenarios. For example, as discussed earlier, venetoclax was identified as a sensitive CYP3A substrate through an interaction study with ketoconazole, a strong CYP3A inhibitor. Due to the large increase in venetoclax exposure, concomitant use of venetoclax with strong CYP3A inhibitors is contraindicated, or venetoclax dose reduction is recommended depending on different treatment phases (FDA, 2016e). When coadministered with moderate CYP3A inhibitors, such as ciprofloxacin, diltiazem, or fluconazole, a 40\%-60\% increase was observed in venetoclax exposure. Considering the risk of toxicities associated with increased exposure, concomitant use of venetoclax with moderate CYP3A inhibitors should also be avoided. If a moderate 
A

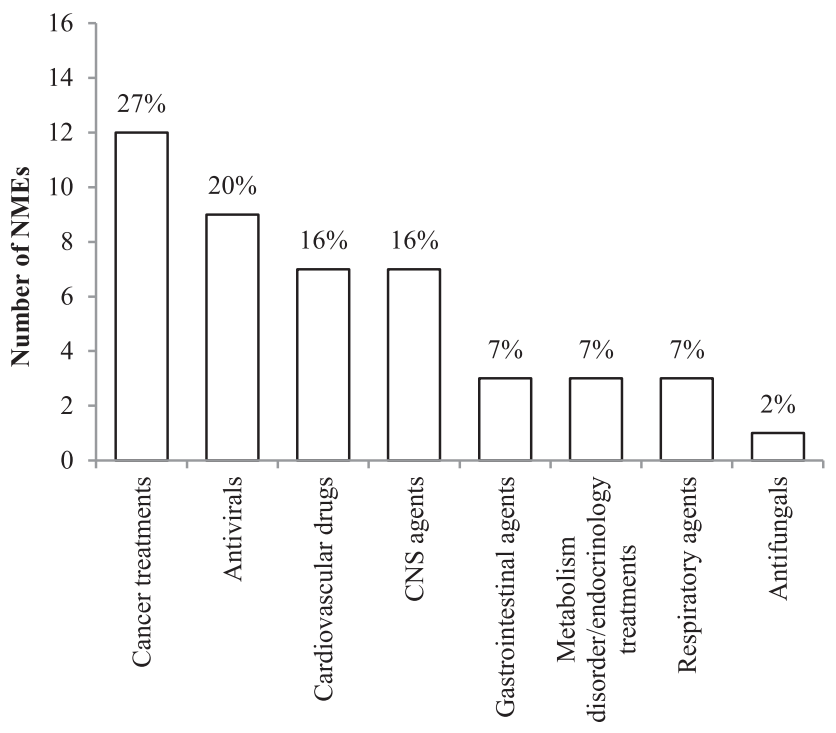

B

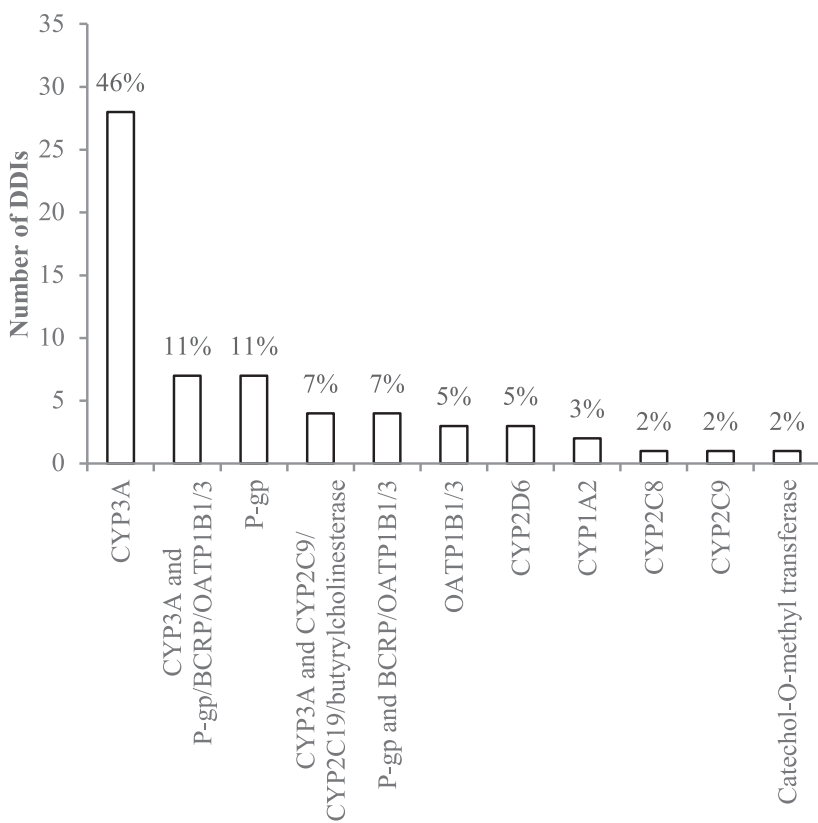

Fig. 1. Quantitation of NMEs acting as substrates in inhibition DDIs for drugs approved by the U.S. FDA between 2013 and 2016 and quantitation of those DDIs. (A) Therapeutic classes of NMEs acting as substrates in inhibition DDIs ( $N=45$ NMEs). The percentage of the total number of NMEs represented in each therapeutic class is shown. (B) Mechanisms of inhibition DDIs with NMEs acting as the substrate ( $N=61$ DDIs). The percentage of the total number of DDIs mediated by each mechanism is shown.

CYP3A inhibitor must be used, the dose of venetoclax should be reduced by at least $50 \%$, and patients need to be monitored closely for signs of toxicities (FDA, 2016e). Exposure to venetoclax was not affected by coadministration of weak CYP3A inhibitors. Additionally, a 600-mg single dose of rifampin increased venetoclax AUC by $78 \%$ and $C_{\max }$ by $113 \%$, likely by inhibiting P-gp-mediated efflux of ventoclax. Labeling recommendations similar to those with moderate CYP3A inhibitors were proposed for concomitant use of venetoclax with P-gp inhibitors (FDA, 2016e).

In Vitro-In Vivo Considerations for NMEs as Substrates. Overall, when all NMEs were evaluated as substrates, CYP3A and $\mathrm{P}$-gp were involved to some degree in approximately $65 \%$ and $30 \%$ of all
A

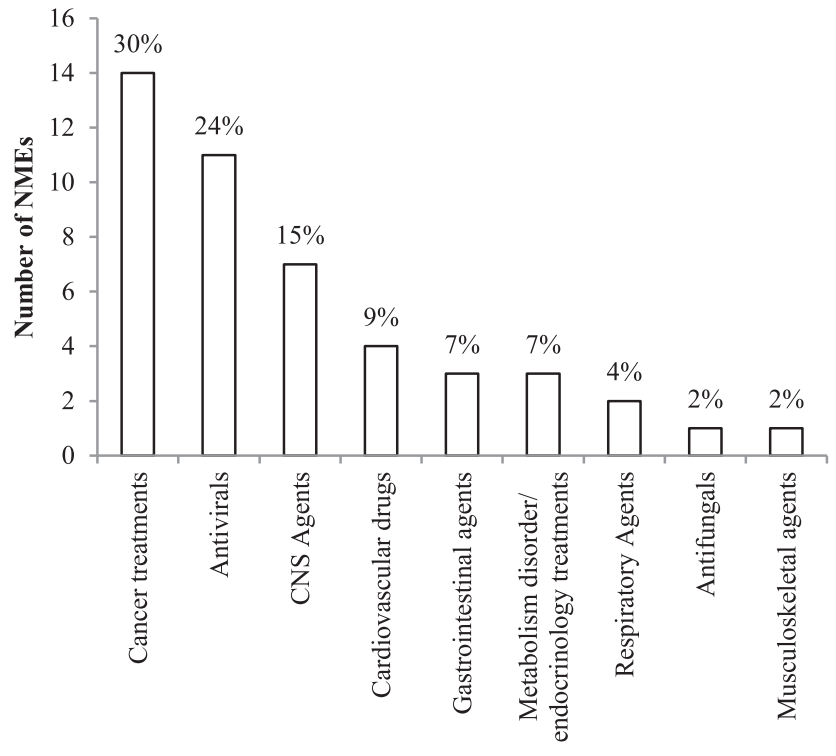

B

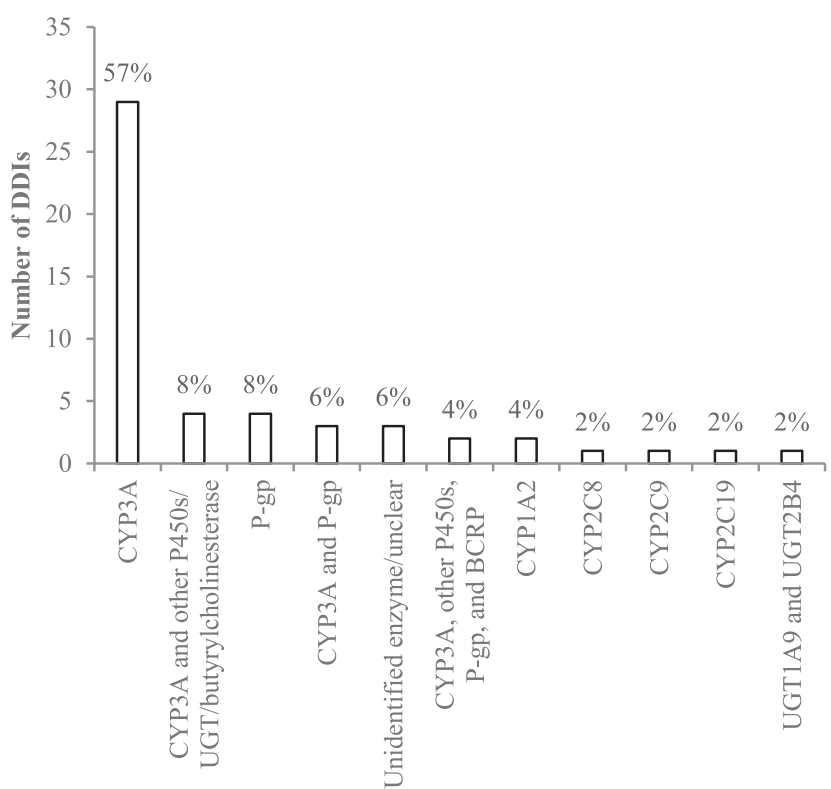

Fig. 2. Quantitation of NMEs acting as substrates in induction DDIs for drugs approved by the U.S. FDA between 2013 and 2016 and quantitation of those DDIs. (A) Therapeutic classes of NMEs acting as substrates in induction DDIs ( $N=46 \mathrm{NMEs})$. The percentage of the total number of NMEs represented in each therapeutic class is shown. (B) Mechanisms of induction DDIs with NMEs acting as the substrate ( $N=51$ DDIs). The percentage of the total number of DDIs mediated by each mechanism is shown.

clinical interactions, respectively. When evaluated in vitro, CYP3A4/5 was shown to metabolize 64 NMEs (Fig. 3A). Of these, 39 NMEs were confirmed in vivo (systemic exposure increases $\geq 25 \%$ ) when coadministered with strong or moderate CYP3A inhibitors. All the drugs with the exception of velpatasvir and netupitant included dosing recommendations in their labeling pertaining to inhibition and/or induction of CYP3A. With regard to P-gp, a total of 47 NMEs were shown to be substrates of P-gp in vitro (more than any other transporter) (Fig. 3B), and $74 \%$ of the clinical CYP3A substrates (29 out of 39 drugs) were shown to be substrates of P-gp in vitro. Twenty-six NMEs were further evaluated in vivo, and 21 showed positive results with AUC ratios of 1.25-7.70. However, among DDIs with large changes of $\geq 5$-fold in victim exposure, the role of P-gp was unclear since the affected drugs were substrates of either CYP3A or OATP1B1/3. 
A

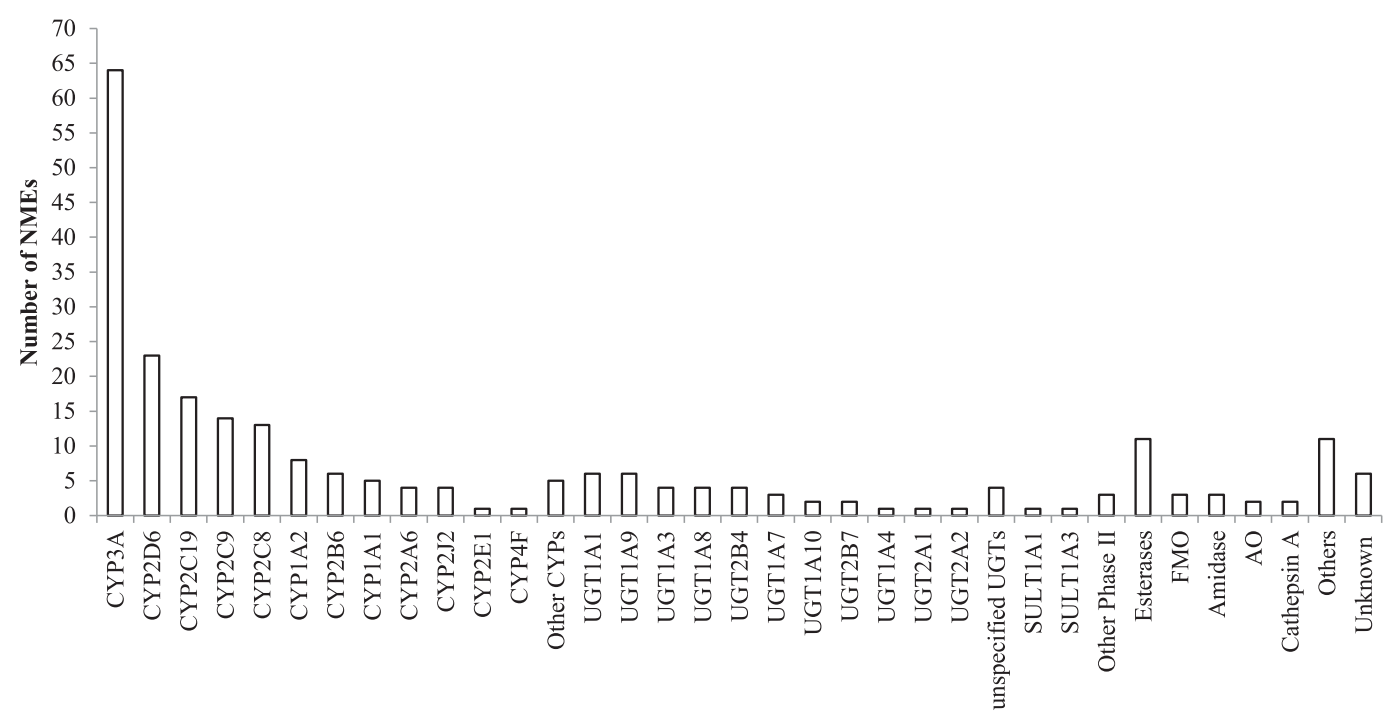

B

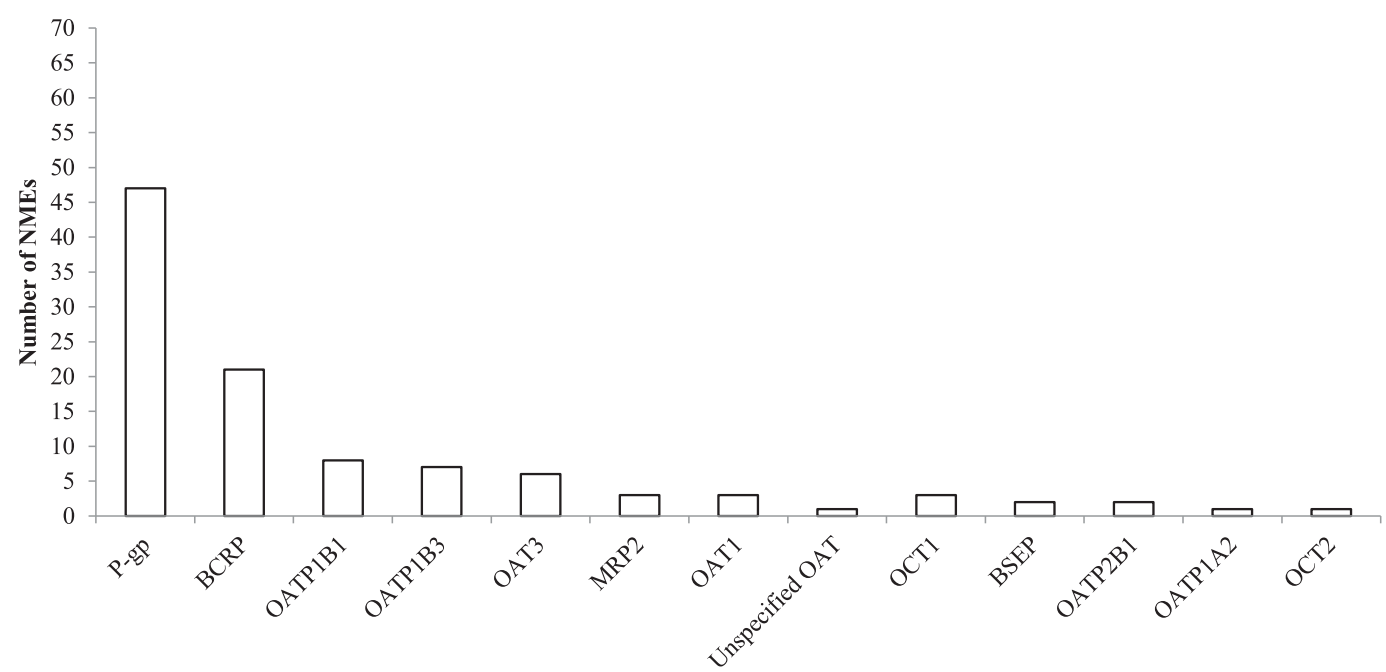

Fig. 3. Quantitation of NMEs acting as substrates of enzymes or transporters for drugs approved by the U.S. FDA between 2013 and 2016. (A) Drug-metabolizing enzymes contributing to NME metabolism. Only parent drugs as substrates of enzymes are shown. Other CYPs were not specified by the authors; other phase II enzymes include SULT2A1, other sulfotransferases, glutathione $S$-transferases, and unspecified conjugated enzymes; others include catecholamine pathway enzymes, epoxide hydrolase, hydrolases, phospholipidase, phosphatase, proteinase, nucleases, nucleotidase, thymidine phosphorylase, and unspecified biotransformation enzymes. AO, aldehyde oxidase; FMO, flavin-containing monooxygenase. (B) Transporters contributing to NME transport. Only parent drugs as substrates of transporters are shown. BSEP, bile salt export pump.

\section{NMEs as Inhibitors}

When NMEs were evaluated as inhibitors, 20 drugs were found to show clinically relevant inhibition, with approximately 40 DDIs presenting AUC ratios $\geq 2$ and 50 DDIs presenting AUC ratios of $1.25-2$ and triggering dose recommendations. Among these drugs, the most represented therapeutic areas are anti-infective agents $(N=8)$, including six antivirals, one antibacterial, and one antifungal, followed by cancer treatments $(N=4)$, CNS drugs $(N=3)$, gastrointestinal agents $(N=3)$, and metabolism disorder/endocrinology treatments $(N=2)$ (Fig. 4A).

DDIs with AUC Ratios 25: Strong Clinical Inhibitors. Only two drugs, the antiviral FDC drug Viekira Pak (paritaprevir, ritonavir, ombitasvir, and dasabuvir; manufactured by AbbVie Inc., North Chicago, IL) and the kinase inhibitor idelalisib, were found to cause strong inhibition, increasing exposure of victim drugs $\geq 5$-fold
(Supplemental Table 5; Table 3). CYP3A was the only enzyme affected, with partial contribution by P-gp. The largest change in exposure was observed with Viekira Pak (paritaprevir/ritonavir 150/100 mg once daily + ombitasvir $25 \mathrm{mg}$ once daily + dasabuvir $400 \mathrm{mg}$ twice daily for 28 days), showing a drastic increase in tacrolimus exposure with an AUC ratio of 57.07. Similarly, an approximately 5-fold increase in cyclosporine (a CYP3A and P-gp substrate) exposure was observed when coadministered with Viekira Pak. Considering the risks associated with large increases in exposure of tacrolimus and cyclosporine, significant dose adjustment and close monitoring of their blood concentrations are recommended for both immunosuppressants when coadministered with Viekira Pak (FDA, 2014m). Since the strong inhibition by Viekira Pak was caused by ritonavir, which is not an NME, this FDC drug was not counted as a strong inhibitor in this analysis. A larger increase in tacrolimus exposure, 80-fold AUC increase, was 
observed when ritonavir was combined with paritaprevir/ombitasvir or paritaprevir/dasabuvir for 28 days. Idelalisib showed strong inhibition of CYP3A, increasing the AUC of midazolam 5.15-fold. Consequently, coadministration of idelalisib with CYP3A substrates should be avoided (FDA, 2014o), and idelalisib is considered a strong inhibitor of CYP3A.

DDIs with $2 \leq$ AUC Ratios < 5: Moderate Clinical Inhibitors. When NMEs served as inhibitors, a total of 36 DDIs showed increases in exposure of victim drugs of 2- to 5-fold perpetrated by 12 drugs (including FDC drugs, so total NME = 15). Among these, five drugs (including eight NMEs) are antivirals (Supplemental Fig. 3A). Detailed DDI data are presented in Supplemental Table 5. In brief, transporters including BCRP, OATP1B1/3, and P-gp seem to play an important role, mediating half of the interactions (Supplemental Fig. 3B). However, due to a lack of substrate specificity, many interactions cannot be attributed to a specific transporter. CYP3A was involved in the drug interactions of four drugs, either as a single contributor or together with P-gp. In addition to P450 enzymes, UGT1A1 also participated in two drug interactions. It is worth noting that the three antiviral FDC drugs identified as moderate inhibitors (Harvoni (manufactured by Gilead Sciences, Inc., Foster City, CA), Viekira Pak, and Zepatier (manufactured by Merck Sharp \& Dohme Corp., Whitehouse Station, NJ)) presented complex inhibition scenarios because each component itself is a clinical inhibitor of multiple enzymes and/or transporters.

DDIs with $1.25 \leq$ AUC Ratios $<2$ and Triggering Dose Recommendations: Weak Clinical Inhibitors. Compared with the number of drugs that showed strong and moderate inhibition, more drugs showed weak inhibition and triggered dose recommendations. Indeed, from approximately 50 DDI studies, a total of 20 NMEs (including three FDC drugs) showed less than 2-fold increases in exposure of victim drugs, and labeling recommendations were made based on these observations (Supplemental Table 5). The most represented drugs were anti-infective agents, including six antivirals, one antibacterial, and one antifungal (Supplemental Fig. 3C).

Transporters mediated half of these weak interactions, most of them attributable to inhibition of P-gp, followed by OATP1B1/3 (Supplemental Fig. 3D). Increases in plasma exposure of digoxin, a clinical substrate of P-gp and a narrow therapeutic range (NTR) drug, appear to be a major concern for DDIs relevant to inhibition of P-gp. Eight drugs, including daclatasvir, eliglustat, flibanserin, isavuconazonium sulfate (prodrug), rolapitant, simeprevir, suvorexant, and velpatasvir, increased the exposure of coadministered digoxin, with AUC and $C_{\max }$ ratios of 1.251.93. Consequently, it has been recommended to monitor digoxin (and other P-gp substrates with an NTR) concentrations and adverse reactions, and adjust digoxin doses if necessary, upon coadministration with any of these drugs (FDA, 2013i, 2014b,c, 2015a,e,f,o, 2016b). Regarding OATP1B1/3mediated interactions, most involved the 3-hydroxy-3-methylglutaryl-CoA reductase inhibitors atorvastatin, pravastatin, rosuvastatin, and simvastatin as victims. Increased risk of myopathy associated with higher statin concentrations is the main reason triggering labeling recommendations. Dose reduction of statins and close monitoring for statin-associated adverse reactions are recommended for the following drugs: daclatasvir, elbasvir/ grazoprevir, eluxadoline, grazoprevir, simeprevir, and Viekira Pak (FDA, 2013i, 2014m, 2015f,p, 2016f). The second-largest group of DDIs was mediated by CYP3A. For example, midazolam exposure was increased by $58 \%, 43 \%$, and $47 \%$ when coadministered with palbociclib, simeprevir, or suvorexant, respectively. Consequently, a dose reduction is recommended for palbociclib, whereas caution and close monitoring of patients are warranted for simeprevir and suvorexant, when coadministered with sensitive CYP3A substrate with an NTR (FDA, 2013i, 2014b, 2015h). As discussed earlier, isavuconazonium sulfate (prodrug) was identified as a moderate inhibitor of CYP3A, with $103 \%$ and $125 \%$ increases observed in the exposure of coadministered midazolam or tacrolimus, respectively (both sensitive CYP3A substrates). A smaller increase (84\%) was observed in
A

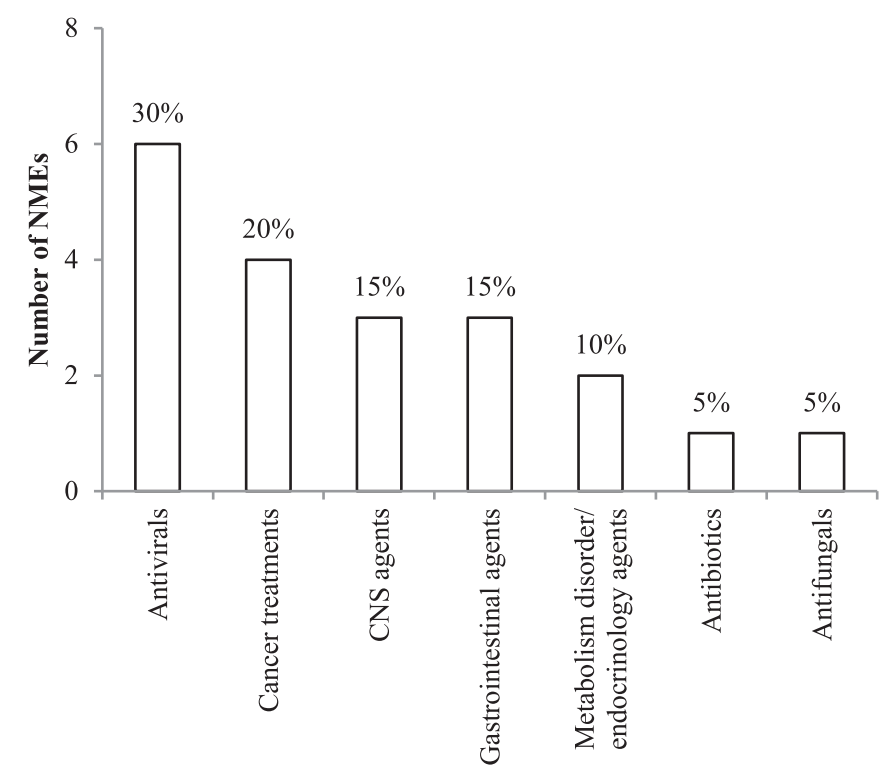

B

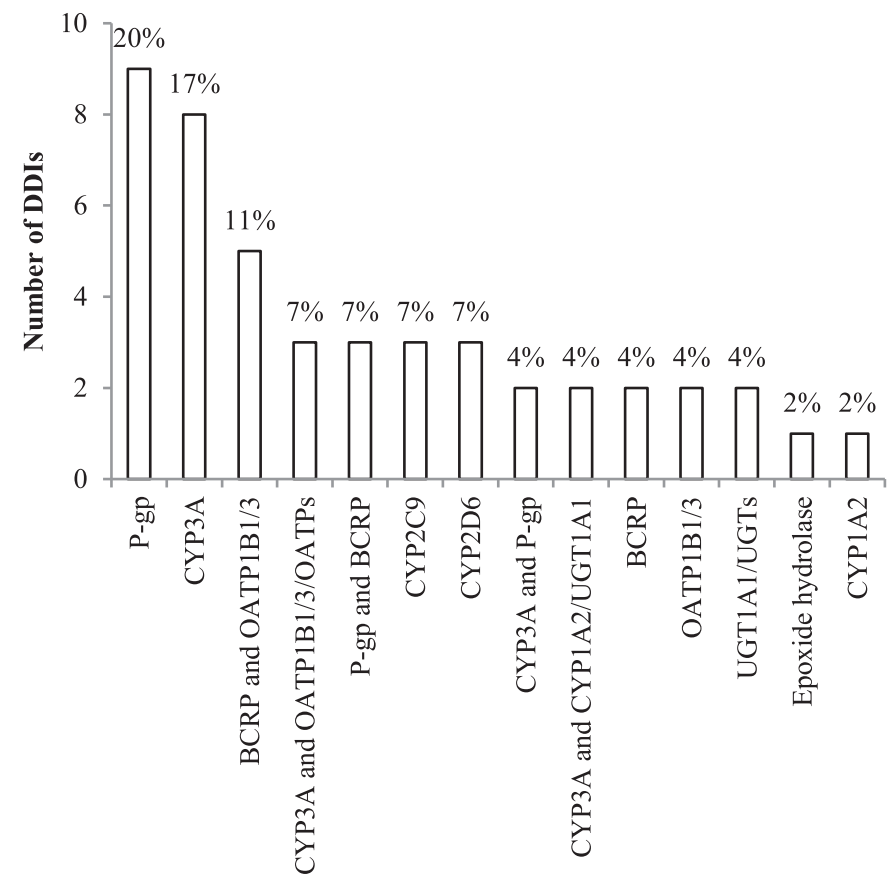

Fig. 4. Quantitation of NMEs acting as perpetrators in inhibition DDIs for drugs approved by the U.S. FDA between 2013 and 2016 and quantitation of those DDIs. (A) Therapeutic classes of NMEs acting as perpetrators in inhibition DDIs $(N=$ 20 NMEs). The percentage of the total number of NMEs represented in each therapeutic class is shown. (B) Mechanisms of inhibition DDIs with NMEs acting as the perpetrator $(N=46 \mathrm{DDI})$. The percentage of the total number of DDIs mediated by each mechanism is shown.

sirolimus exposure (also a sensitive CYP3A substrate), whereas relatively weaker inhibition was observed when it was coadministered with atorvastatin (a moderate sensitive CYP3A substrate) or cyclosporine (a CYP3A substrate with an NTR), with $40 \%$ and $30 \%$ increases in victim drug exposure, respectively.

In Vitro-In Vivo Considerations for NMEs as Inhibitors. Overall, when all NMEs were evaluated as inhibitors, CYP3A and P-gp played a dominant role mediating approximately $60 \%$ (30\% each) of all 
A

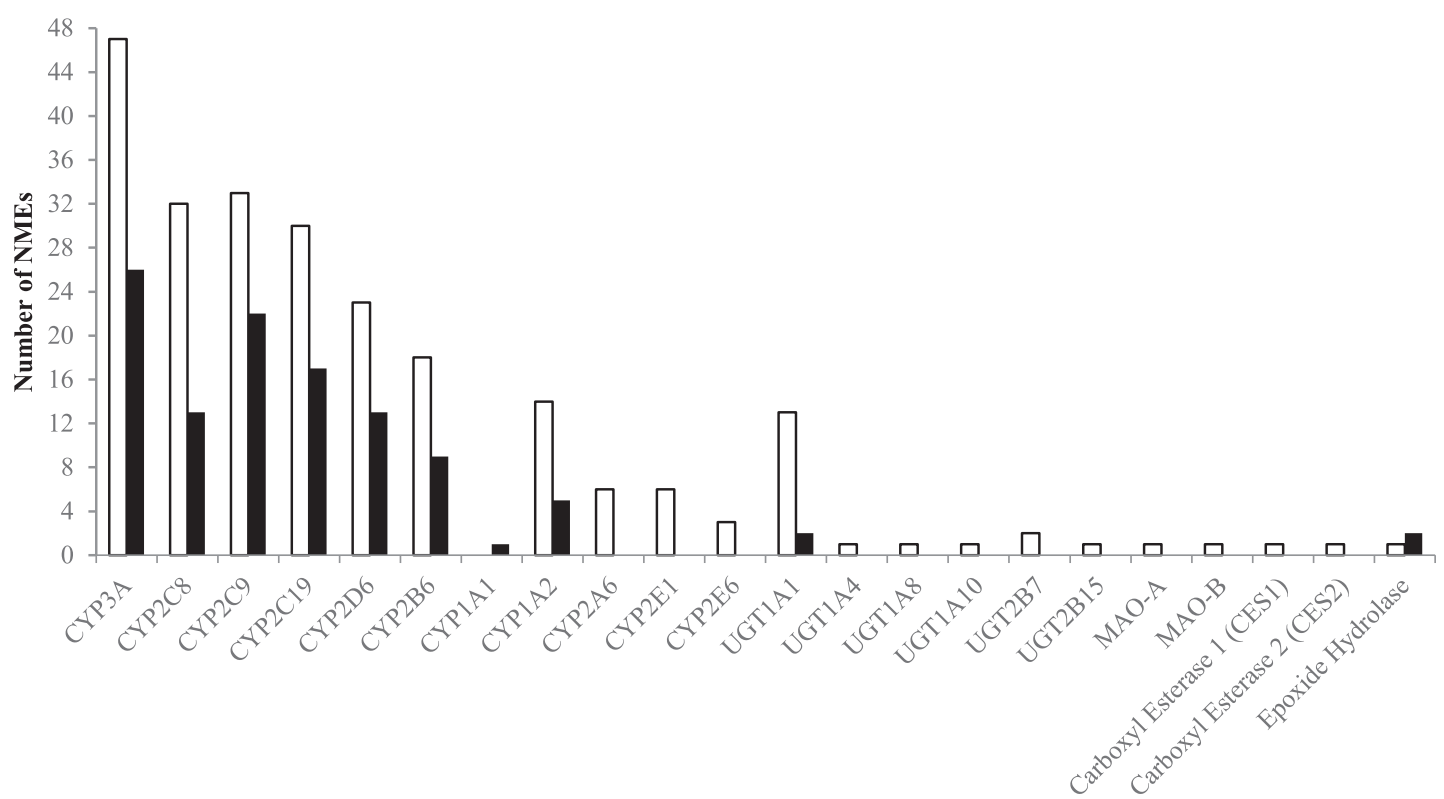

B

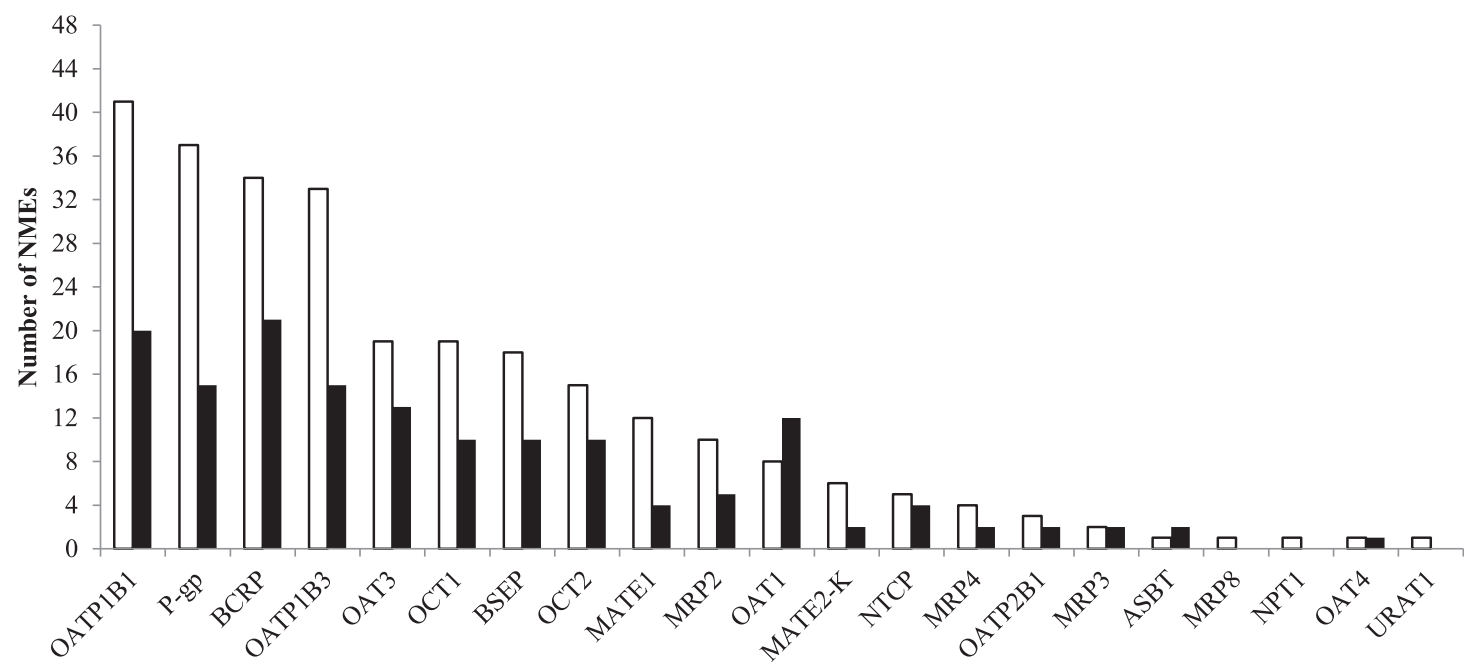

Fig. 5. Quantitation of NMEs acting as inhibitors of enzymes or transporters for drugs approved by the U.S. FDA between 2013 and 2016. (A) Drug-metabolizing enzymes inhibited by NMEs (open bars) and metabolites (closed bars). (B) Transporters inhibited by NMEs (open bars) and metabolites (closed bars). ASBT, apical sodium dependent bile acid transporter; BSEP, bile salt export pump; NTCP, sodium-taurocholate cotransporting polypeptide.

the interactions, followed by OATP1B1/3. As observed in previous years (Yu et al., 2014, 2016, 2017), the majority of the NMEs were extensively evaluated in vitro for their inhibition potential of DMEs and transporters. If an inhibitory effect was observed within the tested concentration range as provided in the NDA reviews, the NME was considered to show positive inhibition in vitro. Following regulatory recommendations described in the FDA draft guidance (FDA, 2012a), an in vitro to in vivo prediction estimate was calculated for major DMEs and transporters. Most drugs with higher $[\mathrm{I}] / \mathrm{IC}_{50},[\mathrm{I}] / \mathrm{K}_{\mathrm{i}}$, or R values than the cutoff were moved forward for clinical evaluations or alternative PBPK simulations. Not surprisingly, CYP3A was the most-often inhibited enzyme in vitro. However, whereas 47 NMEs showed positive inhibition of CYP3A in vitro (Fig. 5A), only 15 drugs (32\%) presented clinical inhibition with $\geq 1.25$-fold increase in the exposure of coadministered CYP3A substrate. With regard to transporters, 41 were in vitro inhibitors of OATP1B1 and 34 were inhibitors of OATP1B3 in vitro (Fig. 5B). When evaluated in vivo, only 10 of these drugs were identified as clinical inhibitors of OATP1B1/3, increasing the exposure of OATP1B $1 / 3$ substrate by $\geq 25 \%$. In terms of P-gp, 37 NMEs were found to inhibit P-gp in vitro (Fig. 5B), and 23 drugs were further evaluated in vivo (including one that was evaluated using PBPK simulations). Only 14 drugs showed positive inhibition in vivo, with $\geq 1.25$-fold increase in the exposure of coadministered P-gp substrate. Likewise, for BCRP, a total of 34 NMEs were found to be inhibitors of BCRP in vitro (Fig. 5B), whereas only 10 were confirmed to inhibit this transporter to a clinically relevant extent, with an AUC ratio $\geq 1.25$ when coadministered with a BCRP substrate. These observations highlight the gap between in vitrobased predictions and clinical evaluation results, since quite a few drugs with a predicted potential risk were not clinically relevant inhibitors, suggesting a need to improve the current prediction models. 
A

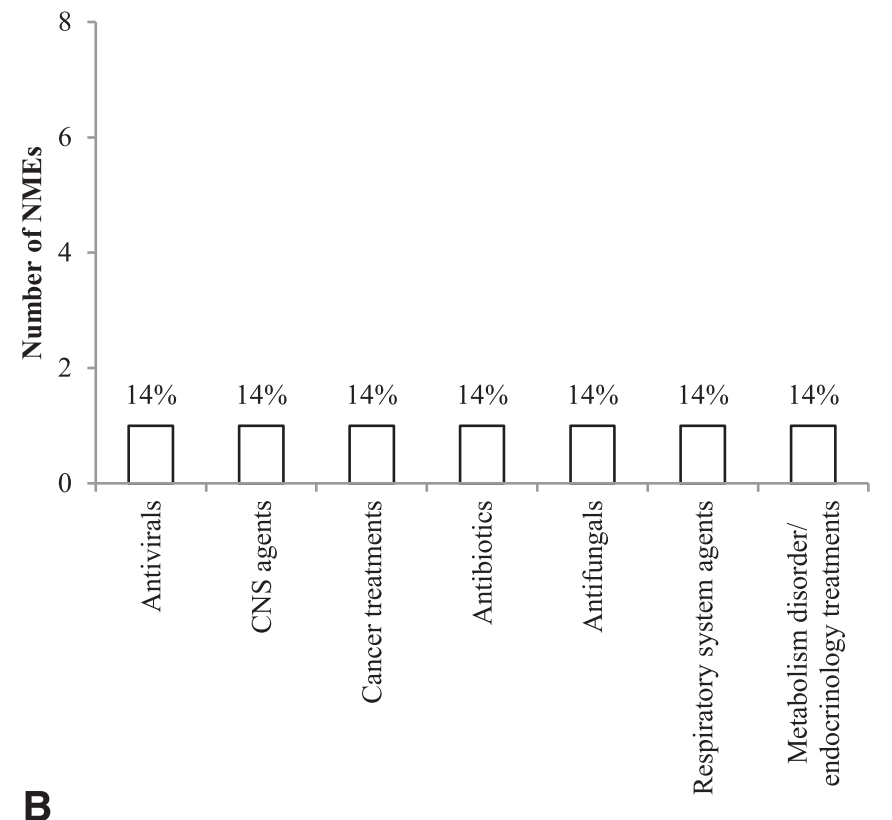

B

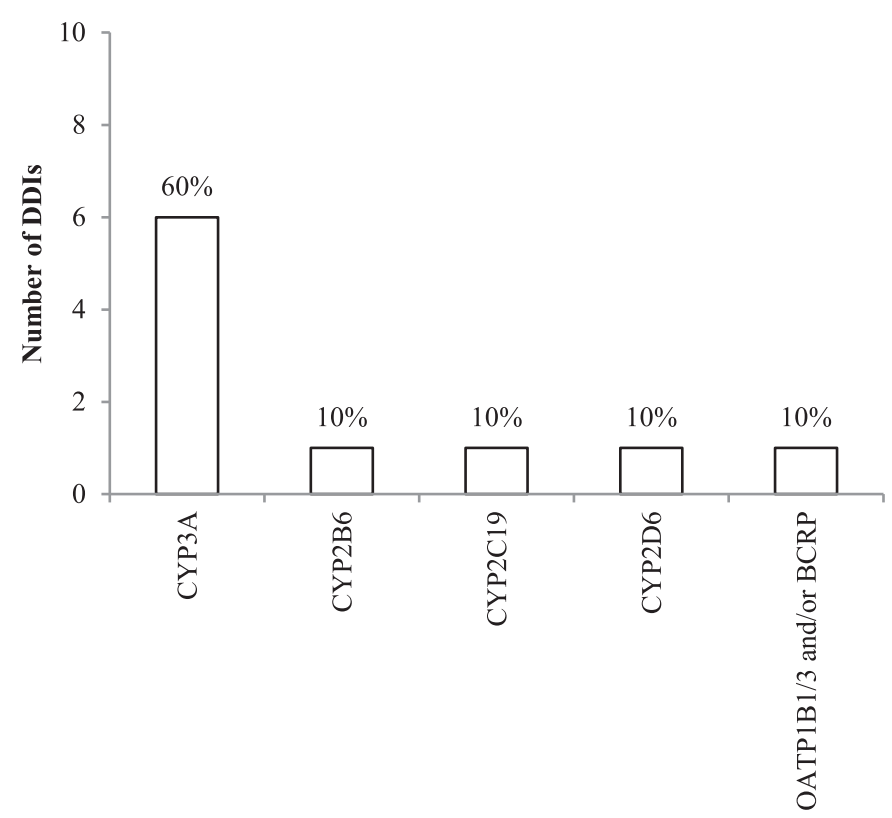

Fig. 6. Quantitation of NMEs acting as perpetrators in induction DDIs for drugs approved by the U.S. FDA between 2013 and 2016 and quantitation of those DDIs. (A) Therapeutic classes of NMEs acting as perpetrators in induction DDIs ( $N=7$ NMEs). The percentage of the total number of NMEs represented in each therapeutic class is shown. (B) Mechanisms of induction DDIs with NMEs acting as the perpetrator ( $N=10$ DDIs). The percentage of the total number of DDIs mediated by each mechanism is shown.

\section{NMEs as Inducers}

As perpetrators, only seven NMEs (including one FDC drug) showed clinically relevant induction (Supplemental Table 6; Table 4). Among them, three drugs are anti-infectives, including one antibacterial (oritavancin), one antifungal (isavuconazonium sulfate), and one antiviral (Viekira Pak) (Fig. 6A). The largest change in victim drug exposure was observed with lumacaftor, which significantly decreased the AUC of ivacaftor, a sensitive CYP3A substrate (FDA, 2012b), by $80 \%$ (lumacaftor and ivacaftor are two components of a combination drug for the treatment of cystic fibrosis). Interestingly, a similar exposure change was observed in itraconazole when it was coadministered with ivacaftor/ lumacaftor (250/200 mg twice daily for 7 days). Based on this observation, coadministration of this combination drug with sensitive CYP3A substrates or CYP3A substrates with an NTR is not recommended. Additionally, hormonal contraceptives (CYP3A substrates) should not be relied upon as an effective method of contraception (FDA, 2015k). The second-largest induction was presented by dabrafenib, reducing the AUC of midazolam by $74 \%$. Consequently, it was noted in the label that concomitant use of dabrafenib with drugs that are sensitive substrates of CYP3A may result in loss of efficacy (FDA, 2013m). In accordance with the FDA guidance (FDA, 2012a), lumacaftor and dabrafenib were identified as strong and moderate CYP3A inducers, respectively. Five drugs, eslicarbazepine acetate, isavuconazonium sulfate, lesinurad, oritavancin, and Viekira Pak (induction mainly caused by ritonavir, which is not an NME), were found to show weak induction (AUC ratios of $0.5-0.8$ ) but still triggered labeling recommendations. Not surprisingly, most of the interactions were mediated by CYP3A. However, induction of other P450s was also observed with the three anti-infective drugs, isavuconazonium sulfate (200 mg once daily), Viekira Pak (paritaprevir/ritonavir 150/100 mg once daily + ombitasvir $25 \mathrm{mg}$ once daily + dasabuvir $250 \mathrm{mg}$ twice daily for 19 days), and oritavancin (1200 mg intravenously), which decreased the AUC of coadministered bupropion (CYP2B6 sensitive substrate), omeprazole (CYP2C19 sensitive substrate), and dextromethorphan (CYP2D6 sensitive substrate) by $42 \%, 38 \%$, and 31\%, respectively (concentration ratio of dextromethorphan to dextrorphan in urine). Interestingly, eslicarbazepine acetate caused a $35 \%$ reduction in rosuvastatin AUC and $C_{\max }$, which maybe attributable to induction of OATP1B 1/3 and/or BCRP. However, there is no in vitro evidence available to fully understand the mechanism.

In vitro evaluation showed that $24 \mathrm{NMEs}$ induced CYP3A, whereas 15 and eight NMEs induced CYP2B6 and CYP1A2, respectively. Activation of the pregnane $\mathrm{X}$ receptor was evaluated for some drugs, and eight NMEs were found to activate this nuclear receptor to some extent (Fig. 7). Dabrafenib, lesinurab, and paritaprevir all showed induction of CYP3A, whereas isavuconazole (the active metabolite of isavuconazonium sulfate) induced both CYP2B6 and CYP3A at clinically relevant concentrations. However, the in vitro enzyme induction potential of eslicarbazepine was not conclusive based on the available data (Bialer et al., 2007; Bialer and Soaresda-Silva, 2012; FDA, 2013c; Zaccara et al., 2015).

\section{Discussion and Conclusion}

A detailed analysis of PK-based DDI data contained in the NDAs approved by the U.S. FDA in the past 4 years (from 2013 to 2016) was performed. Drug interaction profiles and clinical relevance of the outcomes were characterized. CYP3A was confirmed to be a major contributor to clinical DDIs involving NMEs as victims and/or perpetrators, which is consistent with what was found with all the drugs marketed in the past decades. Interestingly, it was found that transporter-based DDIs represented a significant number of all observed drug interactions (about $50 \%$, with NMEs as either victims or inhibitors), although most of these were weakto-moderate interactions. This also reflects the degree of involvement of transporters in DDI evaluations in the past few years.

Overall, when considered as victims, 13 NMEs were identified as sensitive substrates of CYP1A2 (pirfenidone and tasimelteon), CYP2C8 (dasabuvir), CYP2D6 (eliglustat), CYP3A (cobimetinib, ibrutinib, isavuconazole, ivabradine, naloxegol, paritaprevir, simeprevir, and venetoclax), or OATP1B1/3 (grazoprevir), with changes in exposure equal to or greater than 5-fold when coadministered with a strong inhibitor. Among these sensitive substrates, approximately $40 \%$ are anti-infective agents and $22 \%$ are cancer treatment drugs, suggesting a significant risk of clinically relevant DDIs in these patient populations in which therapeutic management is already complex due to 


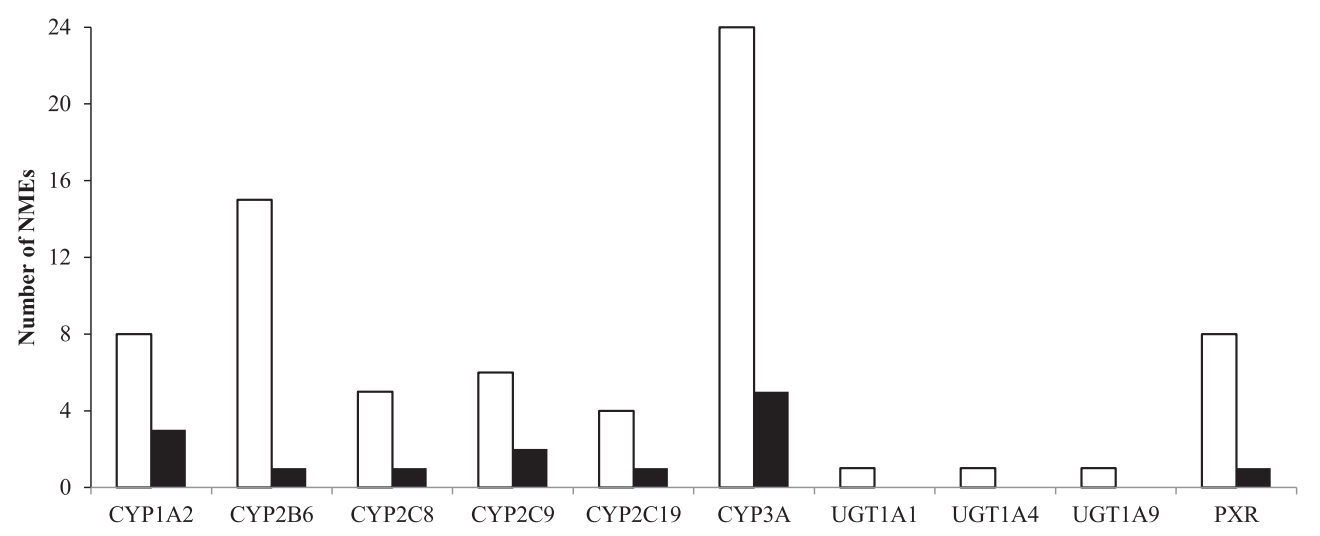

Fig. 7. Quantitation of NMEs acting as inducers of enzymes for drugs approved by the U.S. FDA between 2013 and 2016 . Drug-metabolizing enzymes induced by NMEs (open bars) and metabolites (closed bars) are shown. PXR: pregnane $\mathrm{X}$ receptor.

polytherapy. These two classes of drugs are also the most represented therapeutics approved in the past 4 years, comprising approximately $40 \%$ of all the approved drugs. As expected, approximately $75 \%$ of drugs identified as CYP3A substrates were also substrates of P-gp, consistent with previous findings (Christians et al., 2005; Zhou, 2008). As perpetrators, most clinical DDIs involved weak-to-moderate inhibition or induction, with only one drug (idelalisib) showing strong inhibition of CYP3A, and one NME (lumacaftor) behaving as a strong clinical CYP3A inducer.

Not surprisingly, all the DDIs with exposure changes $\geq 5$-fold in the victim drug were clearly addressed in their labels, mostly as contraindications and coadministration avoidance. There were approximately 125 DDIs with exposure changes (increases or decreases) of 2- to 5-fold with NMEs either as substrates or perpetrators, and over $80 \%$ of these effects triggered dose recommendations in the labels. Interestingly, most of the DDIs that were not reflected in the label pertained to antiviral comedications and were mediated by transporters, such as P-gp and $\mathrm{BCRP}$, functioning as a main or partial factor. For example, coadministration of sofosbuvir with simeprevir, valtapasvir, darunavir/ritonavir + emtricitabine + tenofovir DF, raltegravir + emtricitabine + tenofovir DF, or atazanavir/ritonavir + emtricitabine + tenofovir DF increased the AUC of sofosbuvir 2- to 4-fold. However, considering the safety margins of sofosbuvir, the increase in sofosbuvir exposure was not considered clinically relevant by the sponsor; therefore, no dose adjustment is needed. It is worth noting that approximately 100 DDIs with AUC ratios of 1.25-2 (for inhibition) or $0.5-0.8$ (for induction) resulted in labeling impact, with $52 \%$ related to drugs as substrates, $36 \%$ as inhibitors, and $12 \%$ as inducers. This is understandable because the majority of these interactions were NTR drugs for which small changes in drug exposure may increase the risk of adverse reactions or result in loss of efficacy. The number of DDIs of this group is comparable to that with AUC changes 2- to 5-fold that triggered dose recommendations. Given that a significant number of DDIs with smaller exposure changes triggered label recommendations, special attention should be given to DDIs for NTR drugs. Finally, 14 of the 103 recently approved drugs were combination drugs with highly complex drug interaction profiles in some cases, highlighting the continuous challenge of managing DDIs in clinical practice.

\section{Acknowledgments}

We thank Dr. Sophie Argon, Dr. Katie H. Owens, Dr. Ichiko Petri, Dr. Catherine K. Yeung, and Marjorie Imperial for their contributions to the NDA data curation.

\section{Authorship Contributions}

Participated in research design: Yu, Levy, Ragueneau-Majlessi.

Performed data analysis: Yu, Zhou, Tay-Sontheimer, Levy, RagueneauMajlessi.
Wrote or contributed to the writing of the manuscript: Yu, Zhou, TaySontheimer, Levy, Ragueneau-Majlessi.

\section{References}

Bialer M, Johannessen SI, Kupferberg HJ, Levy RH, Perucca E, and Tomson T (2007) Progres report on new antiepileptic drugs: a summary of the Eigth Eilat Conference (EILAT VIII). Epilepsy Res 73:1-52.

Bialer M and Soares-da-Silva P (2012) Pharmacokinetics and drug interactions of eslicarbazepine acetate. Epilepsia 53:935-946.

Chen G, Lee R, Højer AM, Buchbjerg JK, Serenko M, and Zhao Z (2013) Pharmacokinetic drug interactions involving vortioxetine (Lu AA21004), a multimodal antidepressant. Clin Drug Investig 33:727-736.

Christians U, Schmitz V, and Haschke M (2005) Functional interactions between P-glycoprotein and CYP3A in drug metabolism. Expert Opin Drug Metab Toxicol 1:641-654.

FDA (1996) Drug Approval Package: Allegra (Fexofenadine Hydrochloride). FDA Application NDA 020625, Food and Drug Administration, Silver Spring, MD.

FDA (2012a) Draft Guidance for Industry: Drug Interaction Studies —Study Design, Data Analysis, and Implications for Dosing and Labeling Recommendations, Food and Drug Administration, Silver Spring, MD.

FDA (2012b) Drug Approval Package: Kalydeco (Ivacaftor). FDA Application NDA 203188, Food and Drug Administration, Silver Spring, MD.

FDA (2013a) Drug Approval Package: Adempas (Riociguat). FDA Application NDA 204819, Food and Drug Administration, Silver Spring, MD.

FDA (2013b) Drug Approval Package: Anoro Ellipta (Umeclidinium and Vilanterol). FDA Application NDA 203975, Food and Drug Administration, Silver Spring, MD.

FDA (2013c) Drug Approval Package: Aptiom (Eslicarbazepine Acetate) FDA Application NDA 022416, Food and Drug Administration, Silver Spring, MD.

FDA (2013d) Drug Approval Package: Breo Ellipta (Fluticasone and Vilanterol). FDA Application NDA 204275, Food and Drug Administration, Silver Spring, MD.

FDA (2013e) Drug Approval Package: Brintellix (Vortioxetine). FDA Application NDA 204447, Food and Drug Administration, Silver Spring, MD.

FDA (2013f) Drug Approval Package: Gilotrif (Afatinib). FDA Application NDA 201192, Food and Drug Administration, Silver Spring, MD.

FDA (2013g) Drug Approval Package: Imbruvica (Ibrutinib). FDA Application NDA 205552, Food and Drug Administration, Silver Spring, MD.

FDA (2013h) Drug Approval Package: Invokana (Canagliflozin). FDA Application NDA 204042, Food and Drug Administration, Silver Spring, MD.

FDA (2013i) Drug Approval Package: Olysio (Simeprevir). FDA Application NDA 205123, Food and Drug Administration, Silver Spring, MD.

FDA (2013j) Drug Approval Package: Opsumit (Macitentan). FDA Application NDA 204410 Food and Drug Administration, Silver Spring, MD.

FDA (2013k) Drug Approval Package: Osphena (Ospemifene). FDA Application NDA 203505, Food and Drug Administration, Silver Spring, MD.

FDA (20131) Drug Approval Package: Sovaldi (Sofosbuvir). FDA Application NDA 204671, Food and Drug Administration, Silver Spring, MD.

FDA (2013m) Drug Approval Package: Tafinlar (Dabrafenib). FDA Application NDA 202806, Food and Drug Administration, Silver Spring, MD.

FDA (2013n) Drug Approval Package: Tivicay (Dolutegravir). FDA Application NDA 204790 Food and Drug Administration, Silver Spring, MD.

FDA (2014a) Drug Approval Package: Akynzeo (Netupitant and Palonosetron). FDA Application NDA 205718, Food and Drug Administration, Silver Spring, MD.

FDA (2014b) Drug Approval Package: Belsomra (Suvorexant). FDA Application NDA 204569, Food and Drug Administration, Silver Spring, MD.

FDA (2014c) Drug Approval Package: Cerdelga (Eliglustat). FDA Application NDA 205494, Food and Drug Administration, Silver Spring, MD.

FDA (2014d) Drug Approval Package: Esbriet (Pirfenidone). FDA Application NDA 022535, Food and Drug Administration, Silver Spring, MD.

FDA (2014e) Drug Approval Package: Harvoni (Ledipasvir and Sofosbuvir). FDA Application NDA 205834, Food and Drug Administration, Silver Spring, MD.

FDA (2014f) Drug Approval Package: Hetlioz (Tasimelteon). FDA Application NDA 205677, Food and Drug Administration, Silver Spring, MD.

FDA (2014g) Drug Approval Package: Lynparza (Olaparib). FDA Application NDA 206162, Food and Drug Administration, Silver Spring, MD. 
FDA (2014h) Drug Approval Package: Movantik (Naloxegol). FDA Application NDA 204760, Food and Drug Administration, Silver Spring, MD.

FDA (2014i) Drug Approval Package: Northera (Droxidopa). FDA Application NDA 203202, Food and Drug Administration, Silver Spring, MD.

FDA (2014j) Drug Approval Package: Ofev (Nintedanib). FDA Application NDA 205832, Food and Drug Administration, Silver Spring, MD.

FDA (2014k) Drug Approval Package: Orbactiv (Oritavancin). FDA Application NDA 206334, Food and Drug Administration, Silver Spring, MD.

FDA (20141) Drug Approval Package: Otezla (Apremilast). FDA Application NDA 205437, Food and Drug Administration, Silver Spring, MD.

FDA (2014m) Drug Approval Package: Viekira Pak (Ombitasvir, Paritaprevir, and Ritonavi Co-Packaged with Dasabuvir). FDA Application NDA 206619, Food and Drug Administration, Silver Spring, MD

FDA (2014n) Drug Approval Package: Zontivity (Vorapaxar). FDA Application NDA 204886, Food and Drug Administration, Silver Spring, MD.

FDA (2014o) Drug Approval Package: Zydelig (Idelalisib). FDA Application NDA 206545, Food and Drug Administration, Silver Spring, MD.

FDA (2014p) Drug Approval Package: Zykadia (Ceritinib). FDA Application NDA 205755, Food and Drug Administration, Silver Spring, MD.

FDA (2015a) Drug Approval Package: Addyi (Flibanserin). FDA Application NDA 022526, Food and Drug Administration, Silver Spring, MD.

FDA (2015b) Drug Approval Package: Alecensa (Alectinib). FDA Application NDA 208434, Food and Drug Administration, Silver Spring, MD.

FDA (2015c) Drug Approval Package: Corlanor (Ivabradine). FDA Application NDA 206143 Food and Drug Administration, Silver Spring, MD.

FDA (2015d) Drug Approval Package: Cotellic (Cobimetinib). FDA Application NDA 206192, Food and Drug Administration, Silver Spring, MD.

FDA (2015e) Drug Approval Package: Cresemba (Isavuconazonium Sulfate). FDA Application NDA 207500, Food and Drug Administration, Silver Spring, MD.

FDA (2015f) Drug Approval Package: Daklinza (Daclatasvir). FDA Application NDA 206843, Food and Drug Administration, Silver Spring, MD.

FDA (2015g) Drug Approval Package: Farydak (Panobinostat). FDA Application NDA 207103, Food and Drug Administration, Silver Spring, MD.

FDA (2015h) Drug Approval Package: Ibrance (Palbociclib). FDA Application NDA 207103, Food and Drug Administration, Silver Spring, MD.

FDA (2015i) Drug Approval Package: Ninlaro (Ixazomib Citrate). FDA Application NDA 208462, Food and Drug Administration, Silver Spring, MD.

FDA (2015j) Drug Approval Package: Odomzo (Sonidegib). FDA Application NDA 205266, Food and Drug Administration, Silver Spring, MD.

FDA (2015k) Drug Approval Package: Orkambi (Lumacaftor and Ivacaftor). FDA Application NDA 206038, Food and Drug Administration, Silver Spring, MD.

FDA (2015l) Drug Approval Package: Rexulti (Brexpiprazole). FDA Application NDA 205422, Food and Drug Administration, Silver Spring, MD.

FDA (2015m) Drug Approval Package: Savaysa (Edoxaban). FDA Application NDA 206316, Food and Drug Administration, Silver Spring, MD.

FDA (2015n) Drug Approval Package: Uptravi (Selexipag). FDA Application NDA 207947, Food and Drug Administration, Silver Spring, MD.

FDA (2015o) Drug Approval Package: Varubi (Rolapitant). FDA Application NDA 206500, Food and Drug Administration, Silver Spring, MD.

FDA (2015p) Drug Approval Package: Viberzi (Eluxadoline). FDA Application NDA 206940, Food and Drug Administration, Silver Spring, MD.
FDA (2015q) Drug Approval Package: Vraylar (Cariprazine). FDA Application NDA 204370, Food and Drug Administration, Silver Spring, MD.

FDA (2015r) Drug Approval Package: Yondelis (Trabectedin). FDA Application NDA 207953, Food and Drug Administration, Silver Spring, MD.

FDA (2015s) Drug Approval Package: Zurampic (Lesinurad). FDA Application NDA 207988, Food and Drug Administration, Silver Spring, MD.

FDA (2016a) Drug Approval Package: Briviact (Brivaracetam). FDA Application NDA 205836 Food and Drug Administration, Silver Spring, MD.

FDA (2016b) Drug Approval Package: Epclusa (Aofosbuvir and Velpatasvir). FDA Application NDA 208341, Food and Drug Administration, Silver Spring, MD.

FDA (2016c) Drug Approval Package: Nuplazid (Pimavanserin). FDA Application NDA 207318, Food and Drug Administration, Silver Spring, MD.

FDA (2016d) Drug Approval Package: Ocaliva (Obeticholic Acid). FDA Application NDA 207999, Food and Drug Administration, Silver Spring, MD

FDA (2016e) Drug Approval Package: Venclexta (Venetoclax). FDA Application NDA 208573 Food and Drug Administration, Silver Spring, MD.

FDA (2016f) Drug Approval Package: Zepatier (Elbasvir and Grazoprevir). FDA Application NDA 208261, Food and Drug Administration, Silver Spring, MD.

Hillgren KM, Keppler D, Zur AA, Giacomini KM, Stieger B, Cass CE, and Zhang L; Internationa Transporter Consortium (2013) Emerging transporters of clinical importance: an update from the International Transporter Consortium. Clin Pharmacol Ther 94:52-63.

Huang SM, Strong JM, Zhang L, Reynolds KS, Nallani S, Temple R, Abraham S, Habet SA, Baweja RK, Burckart GJ, et al. (2008) New era in drug interaction evaluation: US Food and Drug Administration update on CYP enzymes, transporters, and the guidance process. $J$ Clin Pharmacol 48:662-670.

Lam YW, Alfaro CL, Ereshefsky L, and Miller M (2003) Pharmacokinetic and pharmacodynamic interactions of oral midazolam with ketoconazole, fluoxetine, fluvoxamine, and nefazodone. $J$ Clin Pharmacol 43:1274-1282.

Mogalian E, German P, Kearney BP, Yang CY, Brainard D, McNally J, Moorehead L, and Mathias A (2016) Use of multiple probes to assess transporter- and cytochrome P450-mediated drug-drug interaction potential of the pangenotypic HCV NS5A inhibitor velpatasvir. Clin Pharmacokinet 55:605-613.

Yu J, Ritchie TK, Mulgaonkar A, and Ragueneau-Majlessi I (2014) Drug disposition and drug-drug interaction data in 2013 FDA new drug applications: a systematic review. Drug Metab Dispos 42:1991-2001

Yu J, Ritchie TK, Zhou Z, and Ragueneau-Majlessi I (2016) Key findings from preclinical and clinical drug interaction studies presented in new drug and biological license applications approved by the Food and Drug Administration in 2014. Drug Metab Dispos 44:83-101.

Yu J, Zhou Z, Owens KH, Ritchie TK, and Ragueneau-Majlessi I (2017) What can be learned from recent new drug applications? A systematic review of drug interaction data for drugs approved by the US FDA in 2015. Drug Metab Dispos 45:86-108.

Zaccara G, Giovannelli F, Cincotta M, Carelli A, and Verrotti A (2015) Clinical utility of eslicarbazepine: current evidence. Drug Des Devel Ther 9:781-789.

Zhou SF (2008) Drugs behave as substrates, inhibitors and inducers of human cytochrome P450 3A4. Curr Drug Metab 9:310-322.

Address correspondence to: Isabelle Ragueneau-Majlessi, Drug Interaction Database Program, Department of Pharmaceutics, University of Washington, Box 357610, Seattle, WA 98195. E-mail: imaj@uw.edu 